Published in final edited form as:

N Engl J Med. 2018 May 17; 378(20): 1951. doi:10.1056/NEJMc1804294.

\title{
Balanced Crystalloids versus Saline in Critically III Adults
}

Matthew W. Semler, MD, MSc ${ }^{1}$, Wesley H. Self, MD, MPH${ }^{2}$, Jonathan P. Wanderer, MD, MPhil $^{3,4}$, Jesse M. Ehrenfeld, MD, MPH ${ }^{3,4,5,6}$, Li Wang, MS ${ }^{7}$, Daniel W. Byrne, MS ${ }^{7}$, Joanna L. Stollings, PharmD, FCCM, FCCP ${ }^{8}$, Avinash B. Kumar, MD, FCCM, FCCP ${ }^{3}$, Christopher G. Hughes, MD, $\mathrm{MSc}^{3}$, Antonio Hernandez, MD, MSc ${ }^{3}$, Oscar D. Guillamondegui, MD, MPH, FACS, FRCPS ${ }^{5}$, Addison K. May, MD, FACS, FCCM ${ }^{5}$, Liza Weavind, MBBCh, FCCM, MMHC ${ }^{3}$, Jonathan D. Casey, MD ${ }^{1}$, Edward D. Siew, MD, MSc ${ }^{9}$, Andrew D. Shaw, MB, FRCA ${ }^{3}$, Gordon R. Bernard, MD ${ }^{1}$, Todd W. Rice, MD, MSc ${ }^{1}$, SMART Investigators ${ }^{*}$, and Pragmatic Critical Care Research Group

${ }^{1}$ Department of Medicine, Division of Allergy, Pulmonary, and Critical Care Medicine, Vanderbilt Center for Kidney Disease (VCKD) and Integrated Program for AKI (VIP-AKI) - all at Vanderbilt University Medical Center, Nashville, TN

${ }^{2}$ Department of Emergency Medicine, Vanderbilt Center for Kidney Disease (VCKD) and Integrated Program for AKI (VIP-AKI) - all at Vanderbilt University Medical Center, Nashville, TN

${ }^{3}$ Department of Anesthesiology, Vanderbilt Center for Kidney Disease (VCKD) and Integrated Program for AKI (VIP-AKI) - all at Vanderbilt University Medical Center, Nashville, TN

${ }^{4}$ Department of Biomedical Informatics, Vanderbilt Center for Kidney Disease (VCKD) and Integrated Program for AKI (VIP-AKI) - all at Vanderbilt University Medical Center, Nashville, TN

${ }^{5}$ Department of Surgery, Vanderbilt Center for Kidney Disease (VCKD) and Integrated Program for AKI (VIP-AKI) - all at Vanderbilt University Medical Center, Nashville, TN

${ }^{6}$ Department of Health Policy, Vanderbilt Center for Kidney Disease (VCKD) and Integrated Program for AKI (VIP-AKI) - all at Vanderbilt University Medical Center, Nashville, TN

${ }^{7}$ Department of Biostatistics, Vanderbilt Center for Kidney Disease (VCKD) and Integrated Program for AKI (VIP-AKI) - all at Vanderbilt University Medical Center, Nashville, TN

Corresponding Author: Todd W. Rice, MD, MSc, todd.rice@vanderbilt.edu, T-1218 MCN, 1161 21st Ave South, Nashville, TN 37232, Phone: 615-322-3412, Fax: 615-343-7448.

Author Contributions: Study concept and design: M.W.S., W.H.S., L.Wa., A.D.S., G.R.B, T.W.R.. Acquisition of data: M.W.S., W.H.S., J.P.W., J.M.E., L.Wa., D.W.B., J.L.S., A.B.K, C.G.H., A.H., O.D.G., A.K.M., L.We., J.D.C., A.D.S., G.R.B, T.W.R.. Analysis and interpretation of data: M.W.S., W.H.S., L.Wa., D.W.B., E.D.S., A.D.S., G.R.B, T.W.R.. Drafting of the manuscript: M.W.S. Critical revision of the manuscript for important intellectual content: M.W.S., W.H.S., J.P.W., J.M.E., L.Wa., D.W.B., J.L.S., A.B.K, C.G.H., A.H., O.D.G., A.K.M., L.We., J.D.C., E.D.S., A.D.S., G.R.B, T.W.R.. Statistical analysis: M.W.S., W.H.S., L.Wa., D.W.B., T.W.R.. Study supervision: M.W.S., W.H.S., A.B.K, C.G.H., A.H., O.D.G., A.K.M., L.We., G.R.B, T.W.R.. M.W.S., L.Wa., and D.W.B. had full access to all the data in the study and take responsibility for the integrity of the data and the accuracy of the data analysis. L.W. and D.W.B. conducted and are responsible for the data analysis.

${ }^{*}$ A full list of the SMART Investigators may be found in the appendix.

Conflicts of Interest: All authors completed and submitted the ICMJE Form for Disclosure of Potential Conflicts of Interest. W.H.S. reported serving on advisory boards for Venaxis Inc, Cempra Pharmaceuticals, Ferring Pharmaceuticals, and BioTest AG, serving as a consultant for Abbott Point of Care, and receiving travel funds from Gilead Pharmaceuticals. A.K.M. reported receiving funding from Atoxbio Ltd. L.We. reported receiving funding from Medtronic Inc. T.W.R. reported serving on an advisory board for Avisa Pharma, LLC and as the Director of Medical Affairs for Cumberland Pharmaceuticals, Inc. 
${ }^{8}$ Department of Pharmaceutical Services, Vanderbilt Center for Kidney Disease (VCKD) and Integrated Program for AKI (VIP-AKI) - all at Vanderbilt University Medical Center, Nashville, TN

${ }^{9}$ Division of Nephrology and Hypertension, Vanderbilt Center for Kidney Disease (VCKD) and Integrated Program for AKI (VIP-AKI) - all at Vanderbilt University Medical Center, Nashville, TN

\section{Abstract}

BACKGROUND: Both balanced crystalloids and saline are used for intravenous fluid administration among critically ill adults. Which results in better clinical outcomes remains unknown.

METHODS: In a pragmatic, cluster-randomized, multiple-crossover trial in five intensive care units at an academic center, we assigned 15,802 adults to receive saline ( $0.9 \%$ sodium chloride) or balanced crystalloids (lactated Ringer's solution or Plasma-Lyte $A \circledR$ ), according to the randomization of the unit to which they were admitted. The primary outcome was Major Adverse Kidney Events within 30 days (MAKE30), i.e., the composite of death, new renal replacement therapy, or persistent creatinine elevation $\geq 200 \%$ of baseline - all censored at the first of hospital discharge or 30 days.

RESULTS: In the balanced crystalloid group, 1,139 patients (14.3\%) experienced MAKE30, compared to 1,211 patients $(15.4 \%)$ in the saline group (marginal odds ratio, $0.91 ; 95 \%$ confidence interval, 0.84-0.99; conditional odds ratio, 0.90; 95\% confidence interval, 0.82-0.99; $\mathrm{P}=0.04$ ). Thirty-day in-hospital mortality was $10.3 \%$ in the balanced crystalloid group and $11.1 \%$ in the saline group $(\mathrm{P}=0.06)$. The incidence of new renal replacement therapy was $2.5 \%$ and $2.9 \%$ respectively $(\mathrm{P}=0.08)$, and the incidence of persistent creatinine elevation was $6.4 \%$ and $6.6 \%$ respectively $(\mathrm{P}=0.60)$.

CONCLUSIONS: Among critically ill adults, the use of balanced crystalloids for intravenous fluid administration appeared to reduce the composite outcome of in-hospital mortality, new renal replacement therapy, and persistent renal dysfunction compared with the use of saline. (SMARTMED and SMART-SURG ClinicalTrials.gov numbers, NCT02444988 and NCT02547779.)

Administration of intravenous crystalloid is common in critical care, yet whether crystalloid composition affects patient outcomes remains unknown. ${ }^{1}$ Historically, $0.9 \%$ sodium chloride (saline) has been the most commonly administered intravenous fluid. ${ }^{2,3}$ Recent data suggest that intravenous saline may be associated with hyperchloremic metabolic acidosis, ${ }^{4}$ acute kidney injury (AKI), ${ }^{5}$ and mortality. ${ }^{6,7}$ Crystalloid solutions with electrolyte compositions closer to that of plasma (balanced crystalloids such as lactated Ringer's solution or PlasmaLyte $A\left({ }^{\circledR}\right)$ represent an increasingly used alternative to saline. ${ }^{8}$ Several observational studies ${ }^{6,9,10}$ and a before-and-after trial ${ }^{5}$ suggested lower rates of acute kidney injury, renal replacement therapy, or death with use of balanced crystalloids. However, two pilot trials ${ }^{11,12}$ found no significant difference between balanced crystalloids and saline in any patient outcome.

To determine the effect of isotonic crystalloid composition on clinical outcomes for critically ill adults, we conducted a pragmatic clinical trial. We hypothesized that use of balanced 
crystalloids would decrease the overall incidence of death, new renal replacement therapy receipt, or persistent renal dysfunction, compared with use of saline.

\section{METHODS}

\section{Study Design and Oversight}

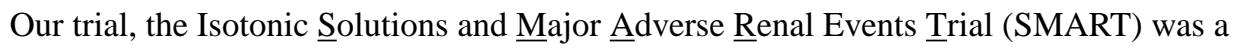
pragmatic, un-blinded, cluster-randomized, multiple-crossover trial comparing balanced crystalloids with saline for intravenous fluid administration among critically ill adults admitted to five intensive care units (ICUs) at Vanderbilt University Medical Center between June 12015 and April 302017.

The trial was approved by the Institutional Review Board at Vanderbilt University with waiver of informed consent (see Supplementary Appendix), was registered online prior to initiation (NCT02444988, NCT02547779), and was overseen by an independent Data and Safety Monitoring Board (DSMB). The study protocol and statistical analysis plan were published prior to the conclusion of enrollment ${ }^{13}$ and may also be found at NEJM.org. The authors designed the trial, collected the data, and performed the analyses. The first author wrote the initial draft of the manuscript. All authors revised the manuscript, vouch for the accuracy and completeness of the data, and approved the decision to submit for publication.

\section{Study Sites and Patient Population}

All adults (age $\geq 18$ years) admitted to a participating ICU during the study period were enrolled at the time of ICU admission (study site characteristics in the Supplementary Appendix). Enrolled patients who were discharged from the hospital were eligible again if they were re-admitted to a participating ICU. We assessed the impact of repeat hospitalizations from unique patients in sensitivity analyses. Patients admitted from the emergency department to a non-ICU ward were enrolled in a separate trial comparing balanced crystalloids and saline among non-critically ill adults, the $\underline{\text { Saline }}$ Against Lactated ringers' or plasmalyTe in the ED (SALT-ED ) trial, reported in this issue of the Journal. ${ }^{14}$

\section{Randomization}

For each month of the trial, participating ICUs were assigned to use either balanced crystalloids or saline for any intravenous isotonic crystalloid administration. ICUs were randomized to use saline during even-numbered months and balanced crystalloid during odd-numbered months, or vice versa (Fig. S1 in the Supplementary Appendix). To allow coordination of crystalloid use between the ICUs and the emergency department and operating rooms, the three ICUs that admit the majority of patients from the emergency department were randomized together, and the two ICUs that admit the majority of patients from the operating rooms were randomized together. ${ }^{13}$ Patients, clinicians, and investigators were not blinded to group assignment.

\section{Study Treatments}

Study protocol determined only the choice of intravenous isotonic crystalloid: $0.9 \%$ sodium chloride (saline group) versus the treating clinician's preference of either lactated Ringer's 
solution or Plasma-Lyte A® (balanced crystalloid group) (Table S1 in the Supplementary Appendix). An advisor within the electronic order entry system informed providers about the trial, asked about relative contraindications to the assigned crystalloid, and, if none were present, guided providers to order the assigned crystalloid. Relative contraindications to the use of balanced crystalloids included hyperkalemia and brain injury. The treating clinician determined the severity of hyperkalemia or brain injury at which saline was used rather than balanced crystalloids. The non-assigned crystalloid was also available from the pharmacy when felt to be required for safe treatment of any patient.

The study was coordinated with the emergency department and operating rooms so that, when feasible, patients being admitted to a participating ICU, or receiving a surgical intervention during the ICU admission, received the crystalloid assigned to that ICU. ${ }^{15}$ Need for access to an intravenous crystalloid at all times precluded use of washout periods, and patients who remained in the ICU from the end of one calendar month to the start of another may have been exposed to both types of crystalloid, the impact of which was evaluated in pre-specified sensitivity analyses.

\section{Data Collection}

We used data collected in routine care and electronically extracted from the electronic health record. ${ }^{12,16}$ Data included pre-enrollment renal function; demographics; diagnoses; predicted risk of in-hospital mortality; orders for intravenous fluids and blood products; plasma electrolyte and creatinine values; receipt of renal replacement therapy; and vital status at hospital discharge. Study personnel blinded to group assignment performed manual chart review to confirm receipt of renal replacement therapy and identify indications for new renal replacement therapy.

\section{Study Outcomes}

The primary outcome was the proportion of patients meeting one or more criteria for Major Adverse Kidney Events within 30 days (MAKE30), ${ }^{16-20}$ the composite of death, new receipt of renal replacement therapy, or persistent renal dysfunction (defined as a final inpatient creatinine value $\geq 200 \%$ of baseline) - all censored at the first of hospital discharge or 30 days after enrollment. The National Institute of Diabetes and Digestive and Kidney Diseases (NIDDK) workgroup on Clinical Trials in AKI recommends MAKE30 as a patient-centered outcome for phase III trials. ${ }^{16,18}$ We determined a value for baseline creatinine using a previously described hierarchical approach prioritizing creatinine measurements in the year prior to hospitalization, then in-hospital measurements prior to ICU admission. Baseline creatinine was estimated using a previously-described three-variable formula when no preenrollment measurements were available (details in the Supplementary Appendix). ${ }^{16,21}$ Patients who had received renal replacement therapy prior to enrollment were ineligible to meet criteria for new renal replacement therapy or persistent renal dysfunction, but could qualify for MAKE30 by experiencing in-hospital mortality.

Secondary clinical outcomes included in-hospital mortality before ICU discharge, 30 days, and 60 days, as well as ICU-free days, ventilator-free days, vasopressor-free days, and renal replacement therapy-free days in the 28 days after enrollment. ${ }^{13}$ Secondary renal outcomes 
included new renal replacement therapy receipt, persistent renal dysfunction, stage II or greater acute kidney injury according to Kidney Disease: Improving Global Outcomes (KDIGO) creatinine criteria, ${ }^{22}$ highest creatinine, change from baseline to highest creatinine, and final creatinine before hospital discharge. ${ }^{13}$

\section{Statistical Analysis}

Complete details of the sample size justification have been published previously. ${ }^{13}$ Initially, we planned to enroll 8,000 patients over 60 unit-months (12 months in 5 ICUs) to detect a $12 \%$ relative difference ${ }^{11,12}$ in MAKE30 between study groups, assuming a $22.0 \%$ incidence of MAKE30 in the saline group based on a prior study. ${ }^{19}$ We subsequently obtained observational data for patients admitted to the study ICUs in the year prior to the trial, which suggested the incidence of MAKE30 in the saline group would be approximately $15.0 \%$. To retain adequate power to detect the targeted relative risk difference, in collaboration with the DSMB, the duration of the trial was increased to 82 unit-months. Enrolling approximately 14,000 patients over 82 unit-months would provide 90 percent statistical power at a type I error rate of 0.05 to detect a $12 \%$ relative difference (1.9\% absolute difference) in MAKE30 between groups. ${ }^{13}$ The DSMB conducted two interim analyses, details of which are in the Supplementary Appendix.

Analyses were conducted at the level of each patient's hospitalization in an intention-to-treat fashion. Continuous variables are reported as mean $\pm \mathrm{SD}$ or median and interquartile range (IQR); categorical variables are reported as frequencies and proportions.

The primary analysis compared MAKE30 between the balanced crystalloid and saline groups using a generalized linear mixed-effects model including fixed effects (group assignment, age, sex, race, source of admission, mechanical ventilation, vasopressor receipt, diagnosis of sepsis, and diagnosis of traumatic brain injury) and random effects (ICU) (Supplementary Appendix). ${ }^{23,24}$ Both the conditional (ICU level) and marginal (population level) effects are reported.

Pre-specified secondary analyses used a similar approach and included-- first, comparison of secondary outcomes between study groups; second, subgroup analyses by study ICU, source of admission, receipt of mechanical ventilation, receipt of vasopressors, sepsis or traumatic brain injury (Supplementary Appendix), baseline renal function, predicted in-hospital mortality, and total volume of isotonic crystalloid through day 30; third, alternative approaches to missing baseline creatinine (Supplementary Appendix); and fourth, sensitivity analyses by volume of crystalloid, accounting for crossover, and limited to each patient's first ICU admission. ${ }^{13}$ Other between-group comparisons were made with the MannWhitney rank-sum test for continuous variables and chi-square test for categorical variables.

A two-sided $\mathrm{P}$ value $<0.048$ indicated statistical significance for the primary outcome after accounting for interim analyses. All other analyses were considered to be hypothesisgenerating. ${ }^{13}$ With 14 secondary outcomes, there was a $51.2 \%$ chance of observing a $\mathrm{P}$ value $<0.05$ for at least one secondary outcome by chance alone. All analyses were performed using R version 3.3.0 software (R Foundation for Statistical Computing, Vienna, Austria) with a pre-specified analysis code published prior to conclusion of enrollment. ${ }^{13}$ 


\section{RESULTS}

\section{Baseline Characteristics}

In all, 15,802 patients were enrolled from 5 ICUs (Fig. S2 in the Supplementary Appendix). Median age was 58 years and $57.6 \%$ of patients were men. More than one-third of patients were receiving mechanical ventilation and one-quarter were receiving vasopressors at enrollment. Patients assigned to balanced crystalloids $(n=7942)$ and saline $(n=7860)$ did not differ at baseline (Table 1, Tables S2-S3 in the Supplementary Appendix).

\section{Fluid Therapy and Electrolytes}

Because fluid therapy in the emergency department and operating room was coordinated with the ICU to which patients were being admitted, the majority of pre-ICU fluid was consistent with study group assignment (Table S4 in the Supplementary Appendix).

The median volume of balanced crystalloid between ICU admission and the first of hospital discharge or 30 days was $1000 \mathrm{~mL}$ [IQR $0-3210 \mathrm{~mL}$ ] in the balanced crystalloid group and the median volume of $0.9 \%$ sodium chloride was $1020 \mathrm{~mL}$ [IQR $0-3500 \mathrm{~mL}$ ] in the saline group (Fig. 1, Tables S5-6 in the Supplementary Appendix). Only 426 patients (5.4\%) in the balanced crystalloid group and 343 patients $(4.4 \%)$ in the saline group were administered any volume of the non-assigned crystalloid as a result of remaining in the ICU from one calendar month to the next (Table S5 in the Supplementary Appendix). The median volume of non-study intravenous fluid, blood products, and medications did not differ between groups (Table S7 in the Supplementary Appendix).

Fewer patients in the balanced crystalloid group than in the saline group had a measured plasma chloride $>110 \mathrm{mmol} / \mathrm{L}(24.5 \%$ vs $35.6 \% ; \mathrm{P}<0.001)$ or a plasma bicarbonate level < $20 \mathrm{mmol} / \mathrm{L}$ (35.2\% vs 42.1\%; P $<0.001$ ) (Fig. 2, Fig. S3 and Table S8 in the Supplementary Appendix). Differences between groups in chloride and bicarbonate concentration were greater for patients with larger volumes of isotonic crystalloid (Figs. S4-5).

\section{Primary Outcome}

A total of 1,139 patients (14.3\%) in the balanced crystalloid group experienced MAKE30, compared with 1,211 patients (15.4\%) in the saline group (marginal odds ratio, $0.91 ; 95 \%$ confidence interval, 0.84 to 0.99 ; conditional odds ratio, $0.90 ; 95 \%$ confidence interval, 0.82 to $0.99 ; \mathrm{P}=0.04$ ) (Table 2, Table S9 and Fig. S6 in the Supplementary Appendix). Results were similar in pre-specified sensitivity analyses: one, restricted to patients with $\geq 500 \mathrm{ml}$ of isotonic crystalloid in the 72 hours after enrollment; two, excluding patients admitted in the week prior to a crossover; three, excluding patients who transferred between ICUs or remained in the ICU through a crossover; four, including only each patient's first ICU admission; five, handling missing baseline creatinine values using imputations, extreme scenarios, or complete cases; and six, using alternative modeling approaches (odds ratios between 0.87 and 0.93 for all sensitivity analyses; Table S10 in the Supplementary Appendix). In pre-specified subgroup analyses, the decrease in MAKE30 with balanced crystalloids compared with saline appeared to be greater among patients administered larger volumes of isotonic crystalloid and patients with sepsis (Fig. 3 and Fig. S7 in the 
Supplementary Appendix). Among patients with sepsis, 30-day in-hospital morality was $25.2 \%$ with balanced crystalloids and $29.4 \%$ with saline (adjusted odds ratio, $0.80 ; 95 \%$ confidence interval, 0.67 to $0.97 ; \mathrm{P}=0.02$ ).

\section{Secondary Outcomes}

In the balanced crystalloid group, 818 patients (10.3\%) died prior to hospital discharge and within 30 days of ICU admission, compared with $875(11.1 \%)$ in the saline group ( $\mathrm{P}=0.06)$ (Table 2, Figs. S8-9 in the Supplementary Appendix). A total of 189 patients (2.5\%) in the balanced crystalloid group and 220 patients $(2.9 \%)$ in the saline group received new renal replacement therapy $(\mathrm{P}=0.08)$ (Table S11 in the Supplementary Appendix). Highest stage of acute kidney injury and incidence of persistent renal dysfunction did not differ between groups (Table 2, Table S12 in the Supplementary Appendix).

\section{DISCUSSION}

Although both saline and balanced crystalloids have been administered to patients in clinical practice for decades, ${ }^{3}$ there are few prior data regarding the effects of crystalloid composition on clinical outcomes. ${ }^{1}$ In pre-clinical models, the high chloride content of saline has been reported to cause hyperchloremia, ${ }^{25}$ acidosis, ${ }^{25}$ inflammation, ${ }^{26}$ renal vasoconstriction, ${ }^{27}$ acute kidney injury, ${ }^{28}$ hypotension, ${ }^{29}$ and death ${ }^{30}$ Studies of healthy volunteers suggest saline may decrease renal perfusion via chloride-mediated renal vasoconstriction. ${ }^{31}$ Observational studies among critically ill adults have reported higher rates of acute kidney injury ${ }^{32}$, renal replacement therapy ${ }^{5,10}$, and death ${ }^{6,7,9,33}$ with use of saline, though results have been inconsistent. ${ }^{34}$ Although underpowered for clinical outcomes, two recent pilot trials among critically ill adults both reported an absolute difference of $1 \%$ in mortality favoring balanced crystalloids. ${ }^{11,12}$

In the current trial, use of balanced crystalloids rather than saline resulted in a $1.1 \%$ absolute reduction in the composite outcome of death, new renal replacement therapy, or persistent renal dysfunction. This finding is consistent with the results of the SALT-ED trial conducted concurrently among non-critically ill adults. ${ }^{14}$ Although modest, the reduction in death, new renal replacement therapy, or persistent renal dysfunction with use of balanced crystalloids may have important implications for the care of the more than 5 million patients admitted to ICUs each year. ${ }^{35}$ These results suggest that using balanced crystalloids rather than saline might prevent one patient from experiencing new renal replacement therapy, persistent renal dysfunction or death for every 94 patients admitted to an ICU. Moreover, the difference in outcomes between balanced crystalloids and saline appeared to be greater for patients with sepsis and patients who received larger volumes of isotonic crystalloid.

The optimal crystalloid composition may depend on the indication for fluid administration and individual patient condition. Concern that the relative hypotonicity of balanced crystalloids might increase intracranial pressure for patients with brain injury led us to systematically present clinicians with the option of administering $0.9 \%$ sodium chloride to patients with brain injury regardless of study group. Our results do not support the safety or efficacy of balanced crystalloids among patients with traumatic brain injury. 
Our study has several strengths. The large sample size provided statistical power to detect small differences in patient outcomes. As in each of the prior trials comparing balanced crystalloids to saline among critically ill adults, $5,11,12$ group assignment in our trial occurred at the level of the ICU. This allowed delivery of the assigned crystalloid early in each patient's critical illness. Enrolling all adults admitted to participating ICUs and allowing clinical providers to deliver the assigned crystalloid within clinical care minimized selection bias and improved generalizability.

Our study has several limitations. Conduct at a single academic center limits generalizability. Treating clinicians were not blinded to composition of the assigned crystalloid or each ICU's group assignment sequence. The mortality and creatinine-based components of the MAKE30 outcome are objective, but a clinician's decision to initiate renal replacement therapy is potentially susceptible to treatment bias. Censoring data collection at hospital discharge may underestimate the true incidence of death by 30 days and may overestimate the true incidence of persistent renal dysfunction at 30 days. ${ }^{16}$ Based on the hypothesized mechanism of chloride-induced organ injury or acidosis, ${ }^{27,31}$ we evaluated lactated Ringer's solution and Plasma-Lyte A® together, and this study does not inform the choice between the two.

In conclusion, in this study of critically ill adults, using balanced crystalloids for intravenous fluid administration, as compared to saline, appeared to decrease the composite outcome of death, new renal replacement therapy, or persistent renal dysfunction.

\section{Supplementary Material}

Refer to Web version on PubMed Central for supplementary material.

\section{ACKNOWLEDGEMENTS}

This trial was conducted within the Vanderbilt Learning Healthcare System. The authors would like to thank the patients, nurses, nurse practitioners, pharmacists, residents, fellows, and attending physicians of the intensive care units at Vanderbilt for making this study possible. In particular, we recognize the mentorship of Arthur P. Wheeler, MD.

Source of Funding: Financial support for the study was provided by the Vanderbilt Institute for Clinical and Translational Research (UL1 TR000445 and UL1TR002243 from NCATS/NIH). M.W.S. was supported in part by the NHLBI (HL087738-09 and K12HL133117). W.H.S was supported in part by the NIGMS (K23GM110469). C.G.H. was supported by American Geriatrics Society Jahnigen Career Development Award and the NIH (HL111111, AG045085, GM120484). A.K.M. was supported in part by the NIH NIGMS (1R01GM115353-01) and the Department of Defense (DoD 12277261). J.D.C. was supported in part by the NHLBI (HL087738-09). E.D.S. was supported by the Vanderbilt Center for Kidney Disease (VCKD). T.W.R. was supported in part by the NIH (R34HL105869). The funding institutions had no role in: conception, design, or conduct of the study; collection, management, analysis, interpretation, or presentation of the data; preparation, review, or approval of the manuscript; or the decision to submit for publication.

\section{REFERENCES}

1. Myburgh JA, Mythen MG. Resuscitation fluids. N Engl J Med 2013;369(13):1243-51. [PubMed: 24066745]

2. Finfer S, Liu B, Taylor C, et al. Resuscitation fluid use in critically ill adults: an international crosssectional study in 391 intensive care units. Crit Care Lond Engl 2010;14(5):R185. 
3. Awad S, Allison SP, Lobo DN. The history of 0.9\% saline. Clin Nutr Edinb Scotl 2008;27(2):17988.

4. Yunos NM, Kim IB, Bellomo R, et al. The biochemical effects of restricting chloride-rich fluids in intensive care. Crit Care Med 2011;39(11):2419-24. [PubMed: 21705897]

5. Yunos NM, Bellomo R, Hegarty C, Story D, Ho L, Bailey M. Association between a chloride-liberal vs chloride-restrictive intravenous fluid administration strategy and kidney injury in critically ill adults. JAMA J Am Med Assoc 2012;308(15):1566-72.

6. Raghunathan K, Shaw A, Nathanson B, et al. Association between the choice of IV crystalloid and in-hospital mortality among critically ill adults with sepsis*. Crit Care Med 2014;42(7):1585-91. [PubMed: 24674927]

7. Rochwerg B, Alhazzani W, Sindi A, et al. Fluid resuscitation in sepsis: a systematic review and network meta-analysis. Ann Intern Med 2014;161(5):347-55. [PubMed: 25047428]

8. Hammond NE, Taylor C, Finfer S, et al. Patterns of intravenous fluid resuscitation use in adult intensive care patients between 2007 and 2014: An international cross-sectional study. PloS One 2017;12(5):e0176292. [PubMed: 28498856]

9. Shaw AD, Raghunathan K, Peyerl FW, Munson SH, Paluszkiewicz SM, Schermer CR. Association between intravenous chloride load during resuscitation and in-hospital mortality among patients with SIRS. Intensive Care Med 2014;40(12):1897-905. [PubMed: 25293535]

10. Shaw AD, Bagshaw SM, Goldstein SL, et al. Major complications, mortality, and resource utilization after open abdominal surgery: 0.9\% saline compared to Plasma-Lyte. Ann Surg 2012;255(5):821-9. [PubMed: 22470070]

11. Young P, Bailey M, Beasley R, et al. Effect of a Buffered Crystalloid Solution vs Saline on Acute Kidney Injury Among Patients in the Intensive Care Unit: The SPLIT Randomized Clinical Trial. JAMA 2015;1-10.

12. Semler MW, Wanderer JP, Ehrenfeld JM, et al. Balanced Crystalloids versus Saline in the Intensive Care Unit. The SALT Randomized Trial. Am J Respir Crit Care Med 2017;195(10):1362-72. [PubMed: 27749094]

13. Semler MW, Self WH, Wang L, et al. Balanced crystalloids versus saline in the intensive care unit: study protocol for a cluster-randomized, multiple-crossover trial. Trials 2017;18(1):129. [PubMed: 28302179]

14. Self WH, Semler MW, Wanderer JP, et al. Balanced Crystalloids versus Saline for Non-critically Ill Adults. N Engl J Med 2018; 378 (9):819-828. [PubMed: 29485926]

15. Self WH, Semler MW, Wanderer JP, et al. Saline versus balanced crystalloids for intravenous fluid therapy in the emergency department: study protocol for a cluster-randomized, multiple-crossover trial. Trials 2017;18(1):178. [PubMed: 28407811]

16. Semler MW, Rice TW, Shaw AD, et al. Identification of Major Adverse Kidney Events Within the Electronic Health Record. J Med Syst 2016;40(7):167. [PubMed: 27234478]

17. Shaw A Models of preventable disease: contrast-induced nephropathy and cardiac surgeryassociated acute kidney injury. Contrib Nephrol 2011;174:156-62. [PubMed: 21921620]

18. Palevsky PM, Molitoris BA, Okusa MD, et al. Design of clinical trials in acute kidney injury: report from an NIDDK workshop on trial methodology. Clin J Am Soc Nephrol CJASN 2012;7(5): 844-50. [PubMed: 22442182]

19. Kashani K, Al-Khafaji A, Ardiles T, et al. Discovery and validation of cell cycle arrest biomarkers in human acute kidney injury. Crit Care Lond Engl 2013;17(1):R25.

20. Kellum JA, Zarbock A, Nadim MK. What endpoints should be used for clinical studies in acute kidney injury? Intensive Care Med 2017;43(6):901-3. [PubMed: 28255614]

21. Zavada J, Hoste E, Cartin-Ceba R, et al. A comparison of three methods to estimate baseline creatinine for RIFLE classification. Nephrol Dial Transplant 2010;25(12):3911-8. [PubMed: 20100732]

22. Kidney Disease: Improving Global Outcomes (KDIGO) Acute Kidney Injury Work Group. KDIGO Clinical Practice Guideline for Acute Kidney Injury. Kidney inter 2012;2(Suppl):8.

23. Parienti J-J, Kuss O. Cluster-crossover design: a method for limiting clusters level effect in community-intervention studies. Contemp Clin Trials 2007;28(3):316-23. [PubMed: 17110172] 
24. Turner RM, White IR, Croudace T, PIP Study Group. Analysis of cluster randomized cross-over trial data: a comparison of methods. Stat Med 2007;26(2):274-89. [PubMed: 16538700]

25. Kellum JA, Bellomo R, Kramer DJ, Pinsky MR. Etiology of metabolic acidosis during saline resuscitation in endotoxemia. Shock Augusta Ga 1998;9(5):364-8.

26. Kellum JA, Song M, Almasri E. Hyperchloremic acidosis increases circulating inflammatory molecules in experimental sepsis. Chest 2006;130(4):962-7. [PubMed: 17035425]

27. Wilcox CS. Regulation of renal blood flow by plasma chloride. J Clin Invest 1983;71(3):726-35. [PubMed: 6826732]

28. Zhou F, Peng Z-Y, Bishop JV, Cove ME, Singbartl K, Kellum JA. Effects of fluid resuscitation with $0.9 \%$ saline versus a balanced electrolyte solution on acute kidney injury in a rat model of sepsis*. Crit Care Med 2014;42(4):e270-278. [PubMed: 24335444]

29. Kellum JA, Song M, Venkataraman R. Effects of hyperchloremic acidosis on arterial pressure and circulating inflammatory molecules in experimental sepsis. Chest 2004;125(1):243-8. [PubMed: 14718447]

30. Kellum JA. Fluid resuscitation and hyperchloremic acidosis in experimental sepsis: improved short-term survival and acid-base balance with Hextend compared with saline. Crit Care Med 2002;30(2):300-5. [PubMed: 11889298]

31. Chowdhury AH, Cox EF, Francis ST, Lobo DN. A randomized, controlled, double-blind crossover study on the effects of 2-L infusions of $0.9 \%$ saline and plasma-lyte ${ }^{\circledR} 148$ on renal blood flow velocity and renal cortical tissue perfusion in healthy volunteers. Ann Surg 2012;256(1):18-24. [PubMed: 22580944]

32. Krajewski ML, Raghunathan K, Paluszkiewicz SM, Schermer CR, Shaw AD. Meta-analysis of high- versus low-chloride content in perioperative and critical care fluid resuscitation. Br J Surg 2015;102(1):24-36. [PubMed: 25357011]

33. Sen A, Keener CM, Sileanu FE, et al. Chloride Content of Fluids Used for Large-Volume Resuscitation Is Associated With Reduced Survival. Crit Care Med 2016;

34. Rochwerg B, Alhazzani W, Gibson A, et al. Fluid type and the use of renal replacement therapy in sepsis: a systematic review and network meta-analysis. Intensive Care Med 2015;41(9):1561-71. [PubMed: 25904181]

35. Wunsch H, Angus DC, Harrison DA, et al. Variation in critical care services across North America and Western Europe. Crit Care Med 2008;36(10):2787-93, e1-9. [PubMed: 18766102]

36. Shahian DM, Wolf RE, Iezzoni LI, Kirle L, Normand S-LT. Variability in the measurement of hospital-wide mortality rates. N Engl J Med 2010;363(26):2530-9. [PubMed: 21175315]

37. Levey AS, Stevens LA, Schmid CH, et al. A new equation to estimate glomerular filtration rate. Ann Intern Med 2009;150(9):604-12. [PubMed: 19414839] 

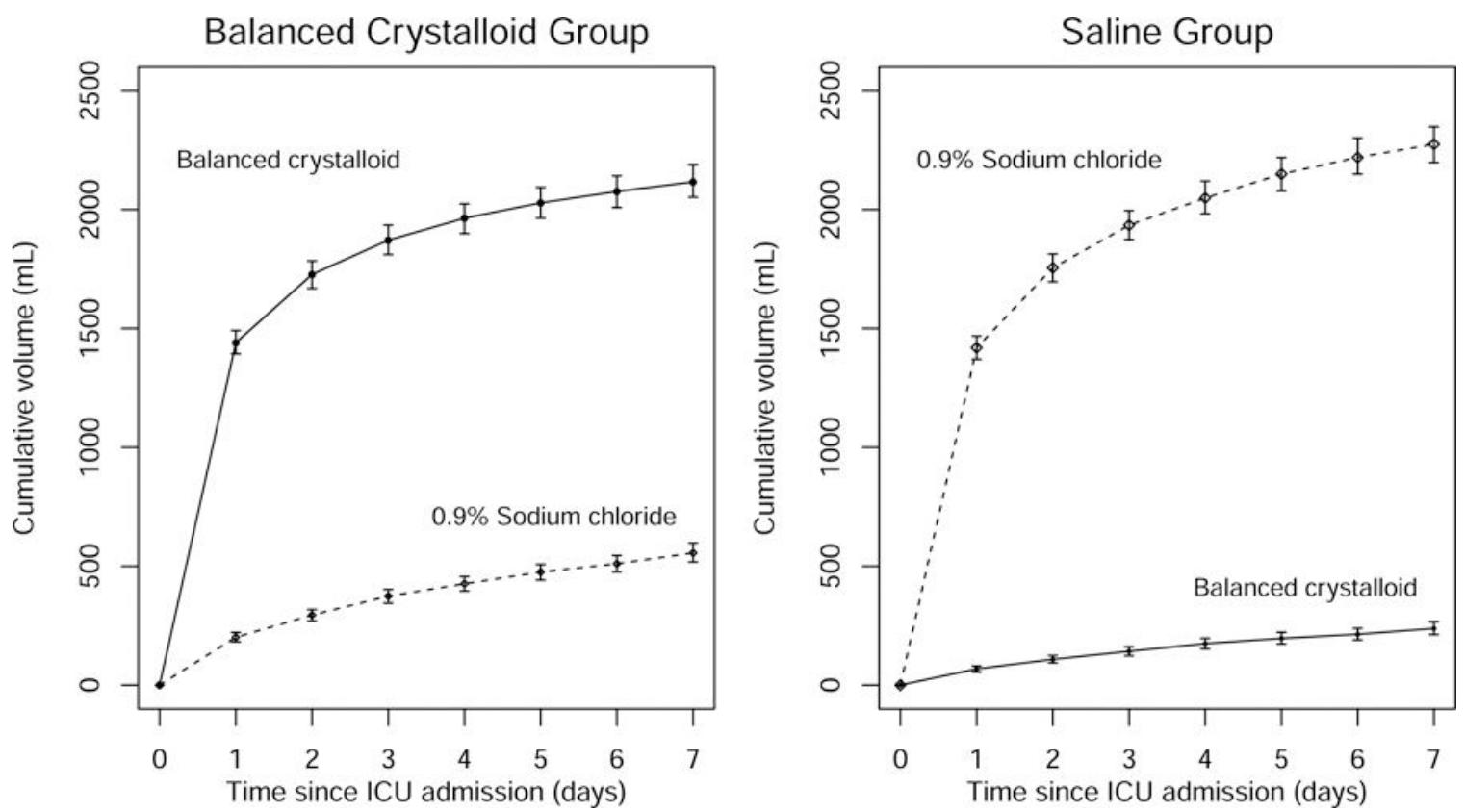

Figure 1. Volume of Intravenous Isotonic Crystalloid by Study Arm.

For patients assigned to the balanced crystalloid group (left) and saline group (right), the cumulative volume (mean and $95 \%$ confidence interval) of intravenous balanced crystalloid and $0.9 \%$ sodium chloride ordered between ICU admission and hospital discharge is displayed over time. 

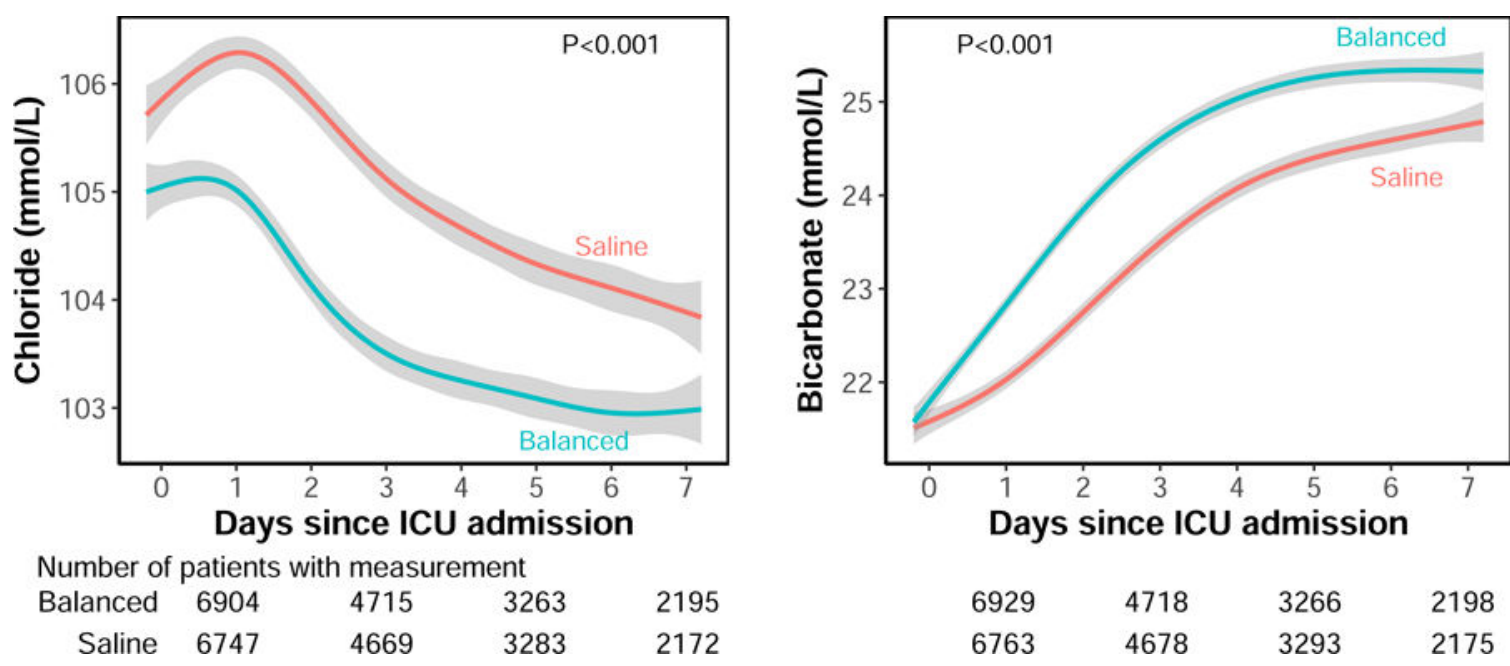

Figure 2. Plasma Chloride and Bicarbonate Concentration by Study Arm.

The mean and $95 \%$ confidence interval for the first plasma chloride (left) or bicarbonate (right) measurement each day are displayed for patients in the balanced crystalloid (blue) and saline (red) groups using locally weighted scatterplot smoothing. Plasma chloride and bicarbonate concentrations were similar between groups at hospital presentation (Table S3 in the Supplementary Appendix), but, because fluid therapy in the emergency department and operating room was coordinated with the ICU to which patients were being admitted, plasma chloride concentration differed between the balanced crystalloid and saline groups at ICU admission. 


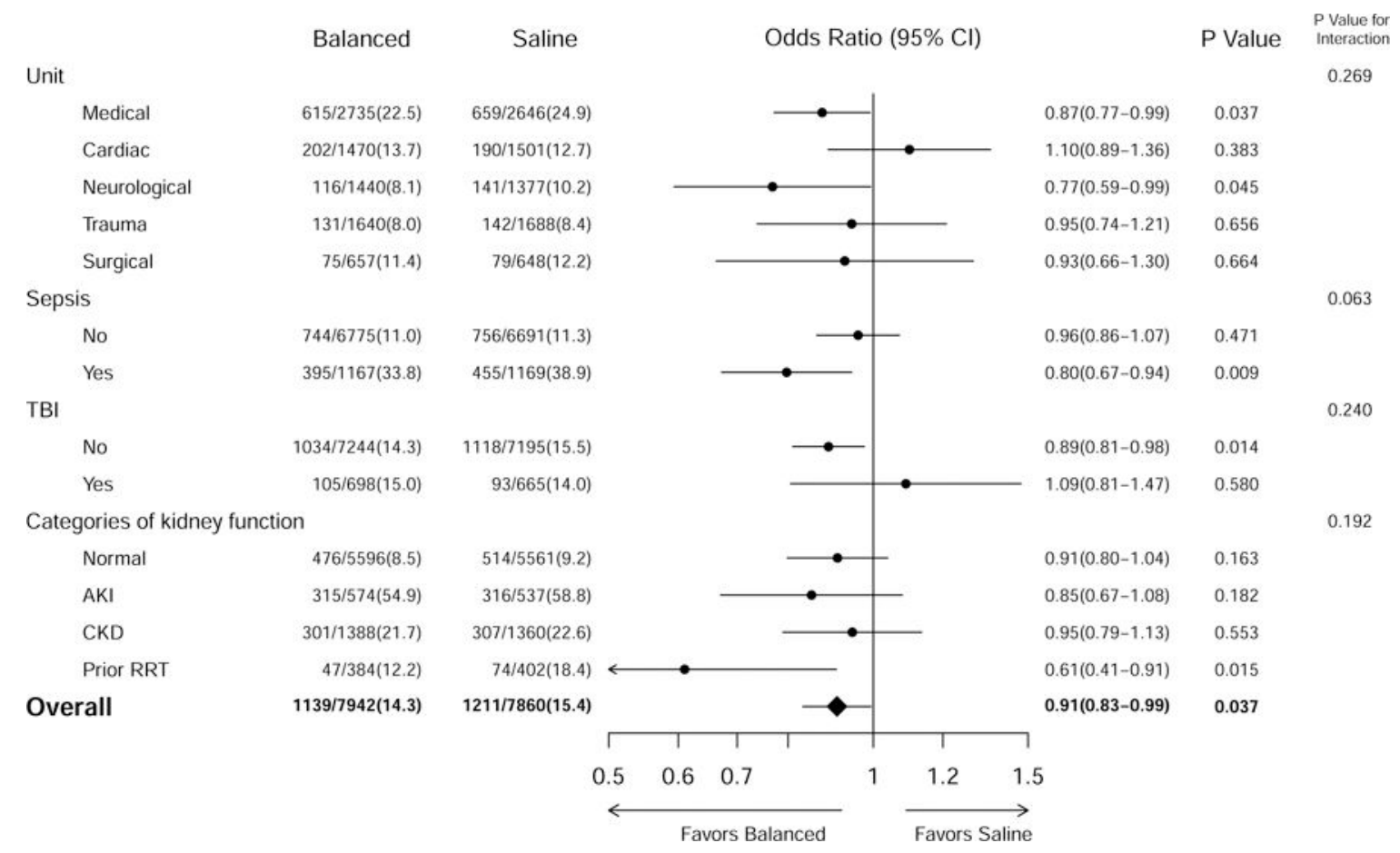

Figure 3. Subgroup Analyses.

For each pre-specified subgroup, the figure displays the number and percentage of patients in each study group who experienced MAKE30, the odds ratio and $95 \%$ confidence interval for experiencing MAKE30 in the balanced crystalloid group compared with the saline group, and the $\mathrm{P}$ values within the subgroup and for the test of interaction derived from a generalized linear mixed-effects model adjusting only for ICU as a random effect (analyses adjusting for additional covariates are displayed in Table 2). TBI is traumatic brain injury. Normal kidney function at enrollment is defined as the absence of acute kidney injury (AKI), chronic kidney disease (CKD), or renal replacement therapy prior to enrollment. AKI refers to patients without CKD whose first creatinine after enrollment was at least $200 \%$ of the baseline value OR both (1) greater than $4.0 \mathrm{mg} / \mathrm{dL}$ and (2) increased at least $0.3 \mathrm{mg} / \mathrm{dL}$ from the baseline value. ${ }^{22} \mathrm{CKD}$ refers to patents with a glomerular filtration rate less than 60 $\mathrm{ml} / \mathrm{min}$ per $1.73 \mathrm{~m}^{2}$ as calculated by the Chronic Kidney Disease Epidemiology (CKD-EPI) Collaboration equation using the patient's baseline creatinine value. ${ }^{37}$ Prior renal replacement therapy (RRT) refers to patients known to have received any form of RRT prior to enrollment. 
Table 1.

Patient Characteristics at Baseline.

\begin{tabular}{|c|c|c|}
\hline Patient Characteristics* & $\begin{array}{c}\text { Balanced } \\
(\mathrm{n}=7942)\end{array}$ & $\begin{array}{c}\text { Saline } \\
(\mathbf{n}=7860)\end{array}$ \\
\hline Age - years & $58[44-69]$ & $58[44-69]$ \\
\hline Men - no. $(\%)$ & $4540(57.2)$ & $4557(58.0)$ \\
\hline Caucasian - no. $(\%)$ & $6384(80.4)$ & $6322(80.4)$ \\
\hline Weight $-\mathrm{kg}^{\dagger}$ & $80[69-96]$ & $79[68-95]$ \\
\hline \multicolumn{3}{|l|}{ Renal comorbidities - no. $(\%)$} \\
\hline Chronic kidney disease, stage III or greater $t^{t}$ & $1388(17.5)$ & $1360(17.3)$ \\
\hline Prior renal replacement therapy receipt & $384(4.8)$ & $402(5.1)$ \\
\hline \multicolumn{3}{|l|}{ Source of admission to the intensive care unit - no. $(\%)$} \\
\hline Emergency department & $3975(50.1)$ & $3997(50.9)$ \\
\hline Operating room & $1732(21.8)$ & $1649(21.0)$ \\
\hline Transfer from another hospital & $1038(13.1)$ & $1018(13.0)$ \\
\hline Hospital ward & $788(9.9)$ & $780(9.9)$ \\
\hline Outpatient & $363(4.6)$ & $359(4.6)$ \\
\hline Another intensive care unit within the hospital & $46(0.6)$ & $57(0.7)$ \\
\hline \multicolumn{3}{|l|}{ Admitting diagnosis - no. $(\%)$} \\
\hline Sepsis or septic shock & $1167(14.7)$ & $1169(14.9)$ \\
\hline Traumatic brain injury & $698(8.8)$ & $665(8.5)$ \\
\hline Mechanical ventilation - no. (\%) & $2723(34.3)$ & $2731(34.7)$ \\
\hline Vasopressors - no. (\%) & $2094(26.4)$ & $2058(26.2)$ \\
\hline Predicted risk of in-hospital mortality $\xi$, mean (95\% CI), \% & $9.4(9.0-9.9)$ & $9.6(9.2-10.0)$ \\
\hline Baseline creatinine $I_{-}-\mathrm{mg} / \mathrm{dL}$ & $0.89[0.74-1.10]$ & $0.89[0.74-1.10]$ \\
\hline Acute kidney injury, stage II or greater"l & $681(8.6)$ & $643(8.2)$ \\
\hline
\end{tabular}

* Continuous data are presented as median $\left[25^{\text {th }}\right.$ percentile $-75^{\text {th }}$ percentile $]$ unless otherwise noted. Categorical data are presented as number (no.) and percentage (\%). There were no significant differences in baseline characteristics between the two study groups (P values range from 0.12 to 0.94$)$.

Information on weight at enrollment was missing for 698 patients.

${ }^{*}$ Chronic kidney disease stage III or greater is defined as a glomerular filtration rate less than $60 \mathrm{ml} / \mathrm{min}$ per $1.73 \mathrm{~m}^{2}$ as calculated by the Chronic Kidney Disease Epidemiology (CKD-EPI) Collaboration equation 37 using the patient's baseline creatinine value.

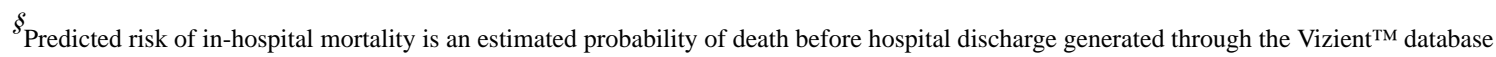
(formerly University HealthSystem Consortium; details at www.vizientinc.com). ${ }^{36}$ Information on predicted risk of in-hospital mortality was missing for 126 patients.

I/ Baseline creatinine for the study is defined as the lowest plasma creatinine measured in the 12 months prior to hospitalization if available, otherwise the lowest plasma creatinine measured between hospitalization and intensive care unit admission; using an estimated creatinine only for patients without an available plasma creatinine between 12 months prior to hospitalization and the time of intensive care unit admission. A total of 863 patients (10.9\%) in the balanced crystalloid group and 826 patients $(10.5 \%)$ in the saline group did not have a measured plasma creatinine value available between 12 months prior to hospital admission and the time of intensive care unit admission (Table S3 in the Supplementary Appendix). 
"Acute kidney injury, stage II or greater is defined according to Kidney Disease Improving Global Outcomes (KDIGO) creatinine criteria 22 as a first plasma creatinine value after enrollment at least $200 \%$ of the baseline value OR both (1) greater than $4.0 \mathrm{mg} / \mathrm{dL}$ and (2) increased at least 0.3 $\mathrm{mg} / \mathrm{dL}$ from the baseline value. 
Table 2.

Clinical Outcomes

\begin{tabular}{|c|c|c|c|c|c|}
\hline Outcome* & $\mathbf{n}$ & $\begin{array}{c}\text { Balanced } \\
(\mathrm{n}=7942)\end{array}$ & $\underset{(n=7860)}{\text { Saline }}$ & $\begin{array}{c}\text { Adjusted } \\
\text { OR }(\mathbf{9 5} \% \mathbf{C I})^{\dagger}\end{array}$ & $\begin{array}{l}\text { Adjusted } \\
P \text { Value }^{\dagger}\end{array}$ \\
\hline \multicolumn{6}{|l|}{ Primary Outcome } \\
\hline Major Adverse Kidney Event within 30 days - no. $(\%)^{\neq}$ & 15802 & $1139(14.3)$ & $1211(15.4)$ & $0.90(0.82-0.99)$ & 0.04 \\
\hline \multicolumn{6}{|l|}{ Components of the Primary Outcome } \\
\hline In-hospital mortality before 30 days & 15802 & $818(10.3)$ & $875(11.1)$ & $0.90(0.80-1.01)$ & 0.06 \\
\hline Receipt of new renal replacement therapy - no. $(\%)^{\xi}$ & 15016 & $189(2.5)$ & $220(2.9)$ & $0.84(0.68-1.02)$ & 0.08 \\
\hline Among survivors & 13444 & $106(1.6)$ & $117(1.8)$ & & \\
\hline Final creatinine $\geq 200 \%$ baseline - no. $(\%)^{\xi}$ & 15016 & $487(6.4)$ & $494(6.6)$ & $0.96(0.84-1.11)$ & 0.60 \\
\hline Among survivors & 13444 & $259(3.8)$ & $273(4.1)$ & & \\
\hline Among survivors without new RRT & 13221 & $215(3.2)$ & $219(3.3)$ & & \\
\hline \multicolumn{6}{|l|}{ Secondary Clinical Outcomes } \\
\hline In-hospital mortality - no. (\%) & 15802 & & & & \\
\hline Before intensive care unit discharge & & $528(6.6)$ & $572(7.3)$ & $0.89(0.78-1.02)$ & 0.08 \\
\hline Before 60 days & & $928(11.7)$ & $975(12.4)$ & $0.92(0.83-1.02)$ & 0.13 \\
\hline Intensive care unit-free days $\mathscr{I}$ & 15802 & $25.3[22.1-26.6]$ & $25.3[22.2-26.6]$ & $1.00(0.89-1.13)$ & 0.94 \\
\hline Mean \pm SD & & $21.8 \pm 8.3$ & $21.7 \pm 8.6$ & & \\
\hline Ventilator-free days $\mathscr{I}$ & 15802 & $28.0[26.0-28.0]$ & $28.0[26.0-28.0]$ & $1.06(0.97-1.16)$ & 0.22 \\
\hline Mean \pm SD & & $24.2 \pm 8.6$ & $23.9 \pm 8.9$ & & \\
\hline Vasopressor-free days $I /$ & 15802 & $28.0[27.0-28.0]$ & $28.0[27.0-28.0]$ & $1.05(0.97-1.14)$ & 0.26 \\
\hline Mean \pm SD & & $24.7 \pm 8.5$ & $24.4 \pm 8.8$ & & \\
\hline Renal replacement therapy-free days $\mathscr{I}$ & 15802 & $28.0[28.0-28.0]$ & $28.0[28.0-28.0]$ & $1.11(1.02-1.20)$ & 0.01 \\
\hline Mean \pm SD & & $25.0 \pm 8.6$ & $24.8 \pm 8.9$ & & \\
\hline \multicolumn{6}{|l|}{ Secondary Renal Outcomes ${ }^{\S}$} \\
\hline $\begin{array}{l}\text { Stage II or greater AKI developing after enrollment - no. } \\
(\%)^{/ /}\end{array}$ & 15016 & 807 (10.7) & $858(11.5)$ & $0.91(0.82-1.01)$ & 0.09 \\
\hline Creatinine ${ }^{* *}, \mathrm{mg} / \mathrm{dL}$ & 14155 & & & & \\
\hline Highest before discharge or day 30 & & $0.99[0.78-1.53]$ & $0.99[0.78-1.52]$ & $1.01(0.97-1.05)$ & 0.58 \\
\hline Change from baseline to highest value & & $0.04[-0.08-0.31]$ & $0.04[-0.08-0.32]$ & $0.98(0.94-1.02)$ & 0.35 \\
\hline Final value before discharge or 30 days & & $0.83[0.70-1.11]$ & $0.83[0.70-1.11]$ & $1.02(0.97-1.06)$ & 0.51 \\
\hline
\end{tabular}

* Continuous data are presented as median $\left[25^{\text {th }}\right.$ percentile $-75^{\text {th }}$ percentile $]$ unless otherwise noted. Categorical data are presented as number (no.) and percentage $(\%)$.

${ }^{\dagger}$ The adjusted odds ratio is for the balanced crystalloid group compared with the saline group. Categorical outcomes are compared between study groups using a generalized linear mixed-effects model adjusting for intensive care unit as a random effect and pre-specified covariates as fixed effects. ${ }^{13}$ Continuous outcomes are compared between groups using a proportional odds model adjusting for the same variables.

NEngl J Med. Author manuscript; available in PMC 2019 June 14. 
FMajor Adverse Kidney Events within 30 days (MAKE30) is the composite of death, receipt of new renal replacement therapy, or final creatinine $\geq$ $200 \%$ baseline, all censored at the first of hospital discharge or 30 days after intensive care unit admission. The effect of study group on MAKE30 shown in the table is the conditional effect. The marginal effect was odds ratio, $0.91 ; 95 \%$ confidence interval, $0.84-0.99$.

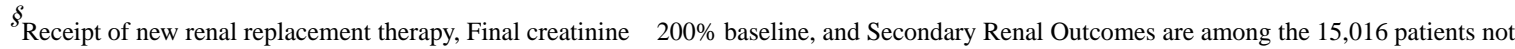
known to have received RRT prior to ICU admission.

\section{ql} Intensive care unit-, ventilator-, vasopressor-, and renal replacement therapy-free days refer to the number of days alive and free from the specified therapy in the first 28 days after enrollment. Odds ratios $\geq 1.0$ indicate a better outcome (i.e., more days alive and free from the specified therapy) with balanced crystalloids compared with saline.

\section{II}

"Stage II or greater acute kidney injury (AKI) developing after enrollment is defined using the Kidney Disease Improving Global Outcomes (KDIGO) creatinine criteria 22 as any creatinine value between enrollment and discharge or 30 days that is (1) increased at least $0.3 \mathrm{mg} / \mathrm{dL}$ from a preceding post-enrollment value and (2) at least $200 \%$ of the baseline value, at least $200 \%$ of a preceding post-enrollment value, or at least 4.0 $\mathrm{mg} / \mathrm{dL}$; or new receipt of renal replacement therapy.

** Among patients who had not received prior renal replacement therapy, plasma creatinine was measured a mean of 8.0 times between enrollment and the first of discharge or 30 days in each group; plasma creatinine was not measured between enrollment and the first of discharge or 30 days for 418 patients $(5.5 \%)$ in the balanced crystalloid group and 443 patients $(5.9 \%)$ in the saline group. 